\title{
SPIN-IT: A DATA CENTRIC ROUTING PROTOCOL FOR IMAGE RETRIEVAL IN WIRELESS NETWORKS
}

\author{
Edward Woodrow and Wendi Heinzelman \\ University of Rochester, Department of Electrical and Computer Engineering \\ Rochester, NY 14627
}

\begin{abstract}
In this paper, we propose and analyze a routing protocol for mobile ad hoc networks that supports efficient image retrieval based on metadata queries. In digital photography, metadata describes captured information about an image and provides the key element needed for advanced techniques for sharing pictures. Our goal was to find an efficient way to utilize metadata to retrieve images in a wireless network of imaging devices. Building on the SPIN protocol for metadata negotiation, we designed SPIN-IT (Sensor Protocols for Information via Negotiation - Image Transfer), a protocol where wireless imaging devices use metadata queries to retrieve desired pictures. This protocol provides low bandwidth query-based communication prior to the transfer of image data to set up routes to desired data rather than routes to specific nodes. We compare SPIN-IT to a centralized approach and discuss the advantages of each design for different picture-sharing scenarios.
\end{abstract}

\section{INTRODUCTION}

Before the advent of digital photography, pictures were generally captured on paper. Technology was developed to produce multiple copies of pictures so they could be shared and reused in various ways. Along with creating, editing, and printing, sharing became one of the fundamental elements of photography, be it personal or professional.

Digital imaging technology has created a desire among users to manage and exchange their images in a variety of ways including storage, e-mail exchange, Internet/WWW postings and other displays (such as personal electronic photo albums or frames), and digital printing. The basis for this explosion of new ways to exploit images is not only the digitization of the image data, but also the inclusion of associated metadata. Metadata is additional data linked to the image data that provides information about the content of the image, the creation of the image, or the uses of the image. Software applications are able to read and interpret this metadata, using it as a parameterization of the image. The International Imaging Industry Association's DIG35 document standardized the "who, what, where, when, why, and how" metadata associated with an image to facilitate the sharing of images among different applications and devices [1].

Wireless networks offer a rich environment for capturing, storing, processing, and sharing data. Future imaging devices connected via a wireless network will be able to exchange not only image data, but also other information, such as environmental data and event-based data. Using metadata, devices will be able to advertise data availability, and query for desired data. These devices will, however, operate within a constrained environment. Energy resources may often be limited, as in the case of small wireless cameras. Similarly, computational power may be minimal. Finally, the wireless network bandwidth and range will likely be constrained.

In this paper we introduce SPIN-IT (Sensor Protocols for Information via Negotiation - Image Transfer), a protocol for sharing images across a wireless network. The protocol is based on the SPIN family of negotiation-based information dissemination protocols [2]. SPIN-IT is designed to conserve energy and bandwidth, while providing users the capability to explore and share pictures within a wireless network. Unlike most protocols designed for ad hoc wireless networks, SPIN-IT is data centric, not node centric - it sets up routes based on data locations rather than node addresses. Thus if two nodes contain the same data, a route to either node is equally valid. This paper describes the design of the SPIN-IT protocol and reports results of simulations that evaluate the protocol's performance, comparing it with a centralized approach to image retrieval.

\section{THE PROTOCOL}

SPIN-IT employs a generalized metadata model, allowing wireless nodes to communicate with each other about what data they want without focusing on the specifics of the data types. This also allows the application to decide what metadata best defines the desired data. The goal has been to perform this exchange as efficiently as possible, conserving energy for the more costly function of transferring the actual image data. 


\subsection{Image Sharing Scenarios}

Each scenario for image sharing has a unique set of requirements relating to the frequency of capture, the advertising of image availability, and the sending of requests for image data. Take, for example, the scenario of a network of security cameras. A likely implementation of such a system might include frequent, scheduled image capture and advertising, and only rare, asynchronous requests for imagery. An ad hoc collection of personal imaging devices at a social gathering, on the other hand, might include random, sporadic image capture along with a comparable set of random, sporadic data requests. These two measures, the rate of capture and the rate of requests for data, represent the protocol overhead for basic route establishment.

These two scenarios may place significantly different traffic on the wireless network depending on the design of the protocol. Both scenarios require a data centric approach to finding desired data, and both require a routing protocol that can identify a stable route for the transfer of large image data sets.

\subsection{SPIN-IT}

SPIN-IT incorporates the dissemination algorithm work done as part of the development of SPIN. In SPIN-IT, queries for specific data are sent to a broadcast address as REQ messages; these REQs are received and processed by all nodes that are within transmission range of the sender. Each node keeps track of the REQ messages it receives using the originating node identifier and a unique request sequence number. New requests are re-broadcast, making SPIN-IT a multi-hop protocol. Nodes use broadcast message suppression by comparing REQ messages with internally queued messages, looking for and deleting redundant messages before rebroadcasting them. Each node adds its source ID to the header of the REQ in order to set up a reverse path route to reach the requesting node.

Once the REQ reaches a node that has the desired data, the node could send the data back to the requesting node using a DATA message. However, in the current implementation of SPIN-IT, source nodes send a ROUTEREPLY message to the requesting node rather than the actual data so that if a requesting node receives more than one reply, the requesting node can choose the "optimal" source, e.g., based on the robustness or perceived longevity of the route. Since SPIN-IT is a data centric routing protocol rather than a node centric routing protocol, a route to any node that has the requested data is equally valid, and the requesting node can use

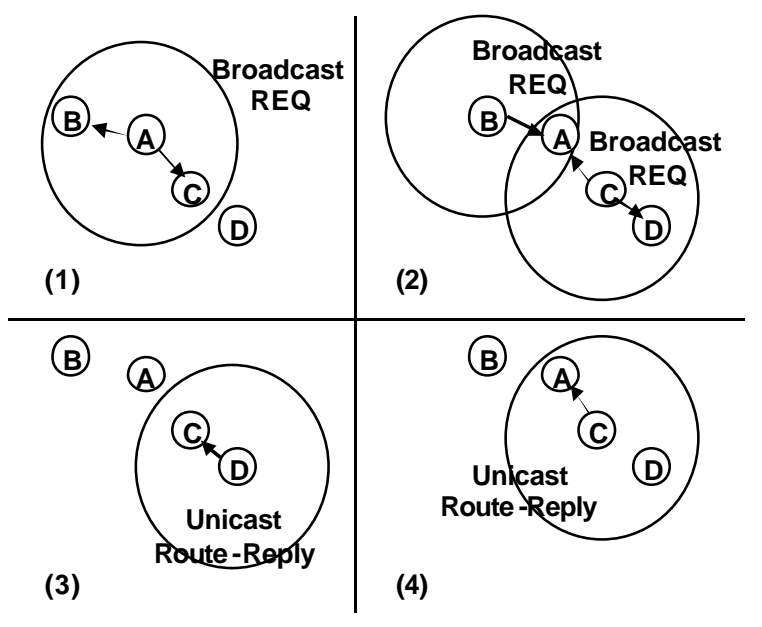

Figure 1. Node A sends out an REQ message for a picture (1). Nodes $\mathrm{B}$ and $\mathrm{C}$ re-broadcast the request (2). Node $\mathrm{D}$, having the requested data, sends a ROUTE-REPLY message back to C (3). Node $\mathrm{C}$ forwards the ROUTE-REPLY message to Node A (4).

information obtained in response to its REQ to choose the best route. The simulations reported in this paper use a simple algorithm similar to Dynamic Source Routing (DSR) [3][4] where the "route record" is maintained in the ROUTE-REPLY packet header and the source closest to the requesting node is chosen for data transfer.

Figure 1 provides a simple illustrative example of an implementation of the SPIN-IT protocol. Node A broadcasts an REQ message indicating, for example, the desire for an image captured around time "2001-0823T13:20:00." Nodes B and C are in range of A and therefore receive the message directly and record the originating node ID and the sequence number (1). Let's assume that neither node possesses the desired image. Nodes B and C would then re-broadcast the request (2). Node A would get the reflected requests and would treat them as ACK messages. Node $\mathrm{D}$, in range of $\mathrm{C}$, would now get the request. In this example, Node $\mathrm{D}$ has the desired data, so it sends a ROUTE-REPLY message to Node C, since it received the request from that node (3). Finally, Node $\mathrm{C}$ would send the ROUTE-REPLY message to Node A as indicated by the path specified in the header of the ROUTE-REPLY message (4).

The route set up for data transfer in the example above is a shortest-path route, as would be found in conventional MANET routing protocols. However, the "data" in SPINIT networks will be a potentially huge buffer of image data. Therefore, shortest-path routing may not be "optimal" for picture-sharing applications. Instead, it may be desirable 
to form routes based on other criterion, such as stability or energy constraints. SPIN-IT is flexible enough to employ different types of routing in conjunction with the REQ messages flooded throughout the network. For example, if stable routes are desired, in order to ensure that there is a high probability that no links in the route will break during data transfer, each node that forwards an REQ could include its current position and velocity vector in the REQ. The source node that has the data can then wait a certain amount of time until it receives several REQs and determine which path listed in the REQ headers represents the most stable path back to the requesting node (the destination). The source node then sends this path to the destination node, using a ROUTE-REPLY message, and data transfer can begin.

The QoS requirements of the picture sharing scenarios will place varying demands on the protocol stack depending on the application. Therefore, the direct request for data will be handled using a protocol that includes a retransmission scheme (e.g., TCP) to ensure correctness of the image data.

\subsection{Alternate Protocols}

An alternative to the distributed SPIN-IT approach to metadata based matching and filtering is to use a centralized directory. In this model, each node establishes and retains a route to a central, coordinating node. The central node maintains a directory of all nodes in the network, their current location, and their associated data. This directory is maintained by having the source nodes push the directory data to the central node using an UPDATE message whenever they obtain new data. $\mathbf{h}$ addition to the metadata of the new data, the UPDATE message contains the source node's current position so that the central directory can maintain a map of current network topology. Nodes seeking data send a request message to the central node, which performs a directory look up and replies to the requesting node with the identification of an appropriate source node and a route to that node.

An example of this model is the Google ${ }^{\mathrm{TM}}$ service on the Internet. This centralized directory is a searchable list of over 1 billion links to network content. While this approach is efficient in a network where the data does not change often, the overhead of keeping track of what data each node possesses may overwhelm a low-bandwidth, energy-constrained wireless network. However, the advantage of a centralized approach is that the overhead in finding the location of desired data is reduced. These trade-offs are similar to those for using a proactive or reactive protocol in MANETs [5].

\section{THE SIMULATION}

In order to study the behavior of the SPIN-IT protocol, and to aid in its development, we created a simulation using the ns software package [6]. ns provides support for the realistic simulation of physical and link layers, the $802.11 \mathrm{~b}$ wireless MAC layer, and node mobility. This framework provided stable, predictable lower network layers, allowing the research to focus on the upper level protocol.

Each node in the network maintains a continually changing list of data elements that it "has" and "wants", i.e., data items it will seek to acquire from other nodes in the network. At random intervals within the simulation, nodes send out request messages (REQs) for data they want to receive. Upon successful receipt of the data, the element is added to the list of items that the node has. Additionally, at random intervals, nodes "capture" entirely new data (as if taking a picture), further increasing the data the nodes posses.

In the set of simulation runs using SPIN-IT, upon receipt of a REQ message, a node performs a Boolean match on the requested data. If it possesses the data, it sends a ROUTE-REPLY message back to the requesting node, including the route in the header of the message. The requesting node can choose the "optimal" route from among the ROUTE-REPLY messages received. In our initial simulations reported here, shortest-path routing is chosen.

\section{SIMULATION RESULTS}

For both the SPIN-IT and centralized models, the total amount of overhead (number of packets generated) during the simulation time was recorded, to determine which approach makes better use of the limited resources under different conditions. For the SPIN-IT model, overhead included the REQ packets flooded through the network in search of a data source and the ROUTE-REPLY messages from the data source(s) to the requesting node. For the centralized model, overhead included the update messages to the central node and the REQ and ROUTE-REPLY message pairs between the requesting nodes and the central node.

To measure protocol performance, we ran similar simulations with both SPIN-IT and the centralized approach and measured the amount of overhead generated by each protocol as we varied the ratio of the rate of capturing new data at each node lambdal and the rate of REQs generated at each node lambda2. Figure 2 shows sample simulation results using a ten-node network, as might be typical for the "social gathering" scenario. We 
see that the crossover point is around 3.7. Therefore, when the number of updates generated throughout the network is greater than 3.7 times the number of REQs generated throughout the network, the SPIN-IT approach produces less overhead per REQ message, whereas when the number of updates is less than 3.7 times the number of REQs, the centralized approach is more efficient.

Our preliminary experimental results show that in largescale networks where there are many more updates than requests, as may occur in highly dynamic networks or networks where new data is continuously generated, SPINIT requires less overhead, allowing more bandwidth to be spent transmitting data and requiring less energy waste than the centralized approach. This might apply to the security camera scenario. However, if the network is fairly static as in our "social gathering" scenario, with few new data being generated and lots of requests for data, a centralized approach is more efficient.

\section{CONCLUSIONS AND FUTURE WORK}

This paper introduced the drivers behind using digital image metadata to facilitate efficient image retrieval in a network of imaging devices and described the preliminary development of protocols that allow devices to obtain images while consuming minimal resources such as bandwidth and energy. Our next goal is to incorporate mobility into our simulations, in order to analyze the performance of the different protocols given realistic network dynamics. For example, as we vary the number of nodes and their mobility patterns, there will be either a large amount of overhead to inform a central node of each node's current location, or the routes in the ROUTEREPLY message from the central node may be stale and a DSR-type route-request may be needed to find a route to the source specified by the central node. Furthermore, we will examine other optimality metrics to incorporate into SPIN-IT's route-finding algorithm, such as route stability or energy constraints along given routes.

We have explored the tradeoffs between a centralized and distributed approach to data management in a picturetaking network, and we found that SPIN-IT is more efficient when the arrival of new data at each node is greater than the arrival of new requests for data in each node. We are currently exploring a hybrid approach to obtain the advantages of both the centralized approach and SPIN-IT for all types of data delivery models. Using a hybrid approach, there are several "central nodes" distributed throughout the network that learn about data from nodes close to them. REQs then only need to pass among these central nodes to find a route to desired data.

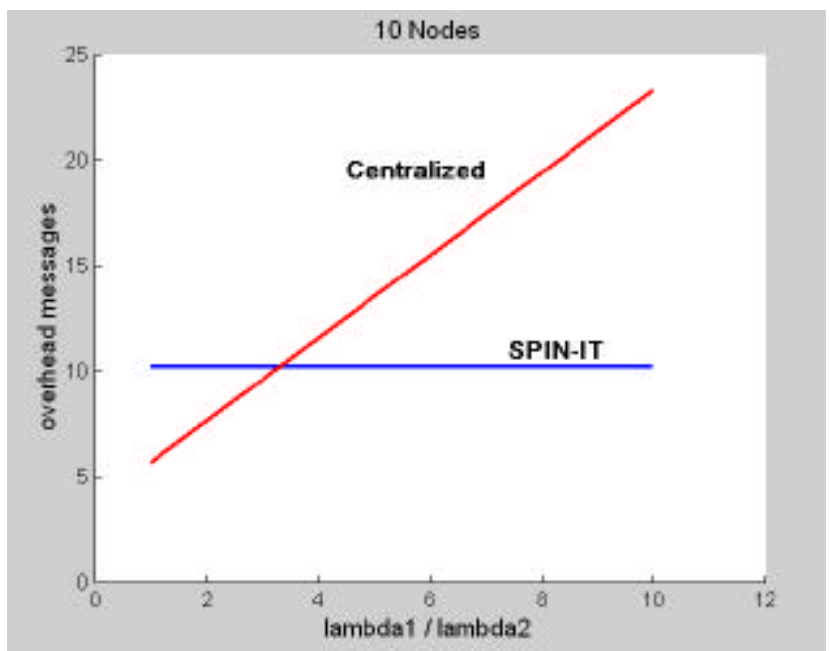

Figure 2 Number of overhead packets per REQ message for a 10-node network as the ratio of UPDATE messages per REQ message is varied. The crossover point occurs at lambda $1 / \operatorname{lambda} 2=3.7$.

Recognizing that a data centric protocol results in the potential availability for several different routes to the same data, we are also exploring the incorporation of multipath routing techniques.

\section{ACKNOWLEDGMENTS}

The authors would like to thank Dr. Charles Judice for his useful suggestions about this work.

\section{REFERENCES}

[1] "DIG35 Specification - Metadata for Digital Images", Version 1.0, Aug., 2000, Digital Imaging Group, Inc.

[2] Heinzelman, W. R., Kulik, J. and Balakrishnan, H., "Adaptive Protocols for Information Dissemination in Wireless Sensor Networks," Proc. Mobicom '99, Seattle, Washington, August 15-20, 1999, pp. 174-185.

[3] Johnson, D. "Routing in Ad Hoc Networks of Mobile Hosts," Proc. IEEE Workshop on Mobile Computing Systems and Applications, pp. 158-163, December 1994.

[4] Chandra, A., Gummalla, V., and Limb, J., "Wireless Medium Access Control Protocols," IEEE Communications Surveys, Second Quarter 2000.

[5] Broch, J, Maltz, D, Johnson, D., Hu, Y. and Jetcheva, J., “A Performance Comparison of Multi-Hop Wireless Ad Hoc Network Routing Protocols," Proc. MobiCom'98, Oct. 1998.

[6] ns-2 Network Simulator, http://www-mash.cs.berkeley.edu /ns, 1998. 